\title{
The burden of hyperkalemia in Germany - a real world evidence study assessing the treatment and costs of hyperkalemia
}

Jennifer Scarlet Haas ${ }^{1 *}$ (D, Kim-Sarah Krinke ${ }^{1}$, Christopher Maas ${ }^{1}$, Thomas Hardt ${ }^{2}$, Isabella Barck ${ }^{2}$ and Sebastian Braun ${ }^{1}$

\begin{abstract}
Background: Hyperkalemia (HK) can affect health outcomes and quality of life, as it is referred to as a potentially life-threatening condition caused by an increased serum potassium concentration in the blood. Patients suffering from heart failure or chronic kidney diseases are at a higher risk of HK, which can further be amplified by the treatment received. To date, data on HK prevalence is lacking for Germany and the aims of this study were to assess HK and compare health-relevant outcomes and healthcare costs between HK patients and non-HK patients.

Methods: The InGef research database containing healthcare claims of over 4 million individuals in Germany was utilized for this retrospective, matched cohort analysis. Patients with non-acute outpatient treated and a subgroup of patients with chronic HK, were identified in 2015 with an individual 1 year pre- and post-index period, taking the first observable HK diagnosis/treatment in 2015 into account as the index event. To identify non-acute outpatient treated HK patients, at least two ICD-10-GM diagnosis codes E87.5 "Hyperkalemia" and/or prescriptions of polystyrene sulfonate were required. Chronic HK patients had additional diagnoses and/or prescriptions in all quarters following the first observable HK diagnosis. Patients without HK were matched 1:1 to the respective HK cohorts.
\end{abstract}

Results: In the year 2015, 3333 patients with non-acute outpatient treated HK were identified of which 1693 were patients with chronic HK. After matching, 3191 and 1664 HK patients and controls were available for analysis. A significantly higher number of hospitalizations was observed for both HK cohorts in comparison to their matched controls. Dialysis initiation as well as the healthcare costs were higher for both HK cohorts when compared to their matched counterparts.

* Correspondence: Jennifer.haas@xcenda.de

'Xcenda GmbH, Lange Laube 31, 30159 Hannover, Germany

Full list of author information is available at the end of the article

(c) The Author(s). 2020 Open Access This article is licensed under a Creative Commons Attribution 4.0 International License, which permits use, sharing, adaptation, distribution and reproduction in any medium or format, as long as you give appropriate credit to the original author(s) and the source, provide a link to the Creative Commons licence, and indicate if changes were made. The images or other third party material in this article are included in the article's Creative Commons licence, unless indicated otherwise in a credit line to the material. If material is not included in the article's Creative Commons licence and your intended use is not permitted by statutory regulation or exceeds the permitted use, you will need to obtain permission directly from the copyright holder. To view a copy of this licence, visit http://creativecommons.org/licenses/by/4.0/ The Creative Commons Public Domain Dedication waiver (http://creativecommons.org/publicdomain/zero/1.0/) applies to the data made available in this article, unless otherwise stated in a credit line to the data. 
(Continued from previous page)

Conclusions: The disease burden was higher for patients with HK, based on a higher proportion of patients with dialysis initiation and higher healthcare costs.

Keywords: Hyperkalemia, Burden of disease, Healthcare costs, Dialysis, Claims data analysis, Matching

\section{Background}

Hyperkalemia (HK) is a potentially life-threatening metabolic condition characterized by an increased serum concentration of potassium. Normal values of potassium have a range from 3.5 to $5 \mathrm{mmol} / \mathrm{l}$, and hyperkalemia is defined by a value of $>=5.1 \mathrm{mmol} / \mathrm{l}[1,2]$; some authors prefer a low limit of $5.5 \mathrm{mmol} / \mathrm{l}[3,4]$.

Clinical symptoms of hyperkalemia are inconstant and unspecific (fatigue, muscular weakness), however, hyperkalemia can cause arrhythmia and heart arrest without pre-occurring clinical signs [5]. The condition of hyperkalemia is usually detected in the context of routine laboratory screening. In the light of possibly lifethreatening consequences, a finding of hyperkalemia implies an urgent demand for medical clarification and treatment [6].

The predominant risk factor of hyperkalemia is advanced chronic kidney disease (CKD). In patients with CKD $\geq 3($ GFR $<60 \mathrm{ml} / \mathrm{min})$, the decreased ability to excrete potassium, especially in combination with diabetes mellitus and/or heart failure frequently results in recurrent and chronic hyperkalemia [7]. Medication (e.g. nonsteroidal anti-inflammatory drugs (NSAID), reninangiotensin-aldosterone system (RAAS)-Inhibitors and lifestyle (irregular nutrition and circadian rhythm) are additional risk factors $[8,9]$.

The true incidence and prevalence of hyperkalemia in the general population is not known, as there are no population-wide studies that examine it [10]. A retrospective analysis based on a selected Canadian population ( $\geq 65$ years) demonstrates that $2.6 \%$ of the individuals from the emergency department and $3.5 \%$ of the hospitalized patients were hyperkalemic [11]. Further retrospective US researches deliver similar incidence rates between 2.5 and $3.2 \%$ [12, 13]. However, due to the low sensitivity, the true frequency in the general population is assumed to be considerably higher.

At present, little is known about the overall non-acute incidence and prevalence of HK in Germany and the related health resource utilization and costs caused by HK. Additionally, recent studies have focused on the occurrence of $\mathrm{HK}$ within a specific disease or within an inpatient setting, rather than on the general prevalence of HK independent of underlying diseases. The differentiation between acute and non-acute HK is important for medical and pharmacoeconomic reasons: Patients with acute HK receive treatment in emergency units following a standardized sequence of membrane stabilization, potassium shift and potassium elimination, usually hemodialysis [14], while patients with non-acute HK usually receive individualized therapy including dietary consulting, medication, and lifestyle adjustment [15]. Therefore, the aims of this claims database analysis were to assess the prevalence of outpatient treated HK and associated comorbidities as well as health-related resource use from the German healthcare perspective. Additionally, health-relevant outcomes as well as healthcare costs between (a) non-acute outpatient treated HK patients and non-HK patients as well as (b) chronic HK patients and non-HK patients in the German Statutory Health System (SHI) were compared.

\section{Methods}

The goal of this analysis was to assess non-acute outpatient treated HK patients and chronic HK patients in terms of health-relevant outcomes. Further, the occurrence of hospitalizations, healthcare costs as well as dialysis initiation in relation to non-HK patients was explored. A retrospective design was applied using claims from the "Institut für angewandte Gesundheitsforschung Berlin" (InGef) research database. The InGef database consists of 6.7 million covered lives from about 60 different health insurances and includes the healthcare resource utilization and costs of services in an anonymized case-by-case individual format. For scientific research projects, an adjusted analysis sample of the InGef database has been drawn, including approximately 4 million covered lives which represent the German population in terms of age and gender (representing $4.8 \%$ of the German population and $5.6 \%$ of the German SHI population). Further, the analysis sample has been proven to have good external validity in terms of morbidity, mortality and drug use [16].

\section{Patient identification}

The complete study period included data from January 1st, 2014 through December 31st, 2016 with an enrollment period from January 1st, 2015 through December 31st, 2015. Selected patients were required to be observable within an individual pre-index period of 4 quarters and an individual post-index period including the index quarter and 3 consecutive quarters. The first observable diagnosis or prescription marked the index event/quarter. 
Non-acute outpatient treated HK patients and those with chronic HK were identified and defined the main cohorts of interest. To that end, all patients who had at least one inpatient or outpatient ICD-10-GM (International Statistical Classification of Diseases and Related Health Problems, 10th revision, German Modification) diagnosis code of E87.5 "Hyperkalemia" or at least one prescription of polystyrene sulfonate (ATC code: V03AE01), were identified in 2015.. As a next step, we excluded all patients who were $<18$ years in the quarter of their index event. Out of these patients, patients with non-acute outpatient treatment for hyperkalemia (cohort 1a) were identified by having at least two HK diagnoses and/or polystyrene sulfonate prescriptions with a restriction to one inpatient diagnosis and a follow-up outpatient diagnosis, indicating non-acute HK treatment.. Out of the cohort of non-acute outpatient treated HK patients, the subgroup of chronic HK patients (cohort 1b) was identified. These patients were required to have outpatient HK diagnoses or polystyrene sulfonate prescriptions in each quarter of the follow-up period. Figure 1 presents an overview of the steps that were performed to identify the respective HK patient cohorts as well as the respective cohort without HK from the database.

\section{Matching approach}

Individuals without hyperkalemia (cohort $2 \mathrm{a} / 2 \mathrm{~b}$ ) were matched 1:1 to each identified hyperkalemia patient of cohort 1a and cohort $1 \mathrm{~b}$. Therefore, patients without any diagnosis of $\mathrm{HK}$ and without any prescription of polystyrene sulfonate during the whole study period (January 1st, 2014 to December 31st, 2016) were identified from the InGef database (cohort $2 \mathrm{a} / 2 \mathrm{~b}$ ). Index quarters were assigned to adult patients without hyperkalemia based on the distribution of the index quarters in the cohorts 1a and 1b. An exact matching approach without replacement was applied for both matchings of the cohorts $1 \mathrm{a}$ and $2 \mathrm{a}$ and $1 \mathrm{~b}$ and $2 \mathrm{~b}$, respectively. The matching parameters included age, gender, number of hospitalizations in the index quarter, prescriptions of RAASi in the index quarter (yes/no), and chronic kidney disease with related stages, taking the highest coded stage in the pre-index period into account.

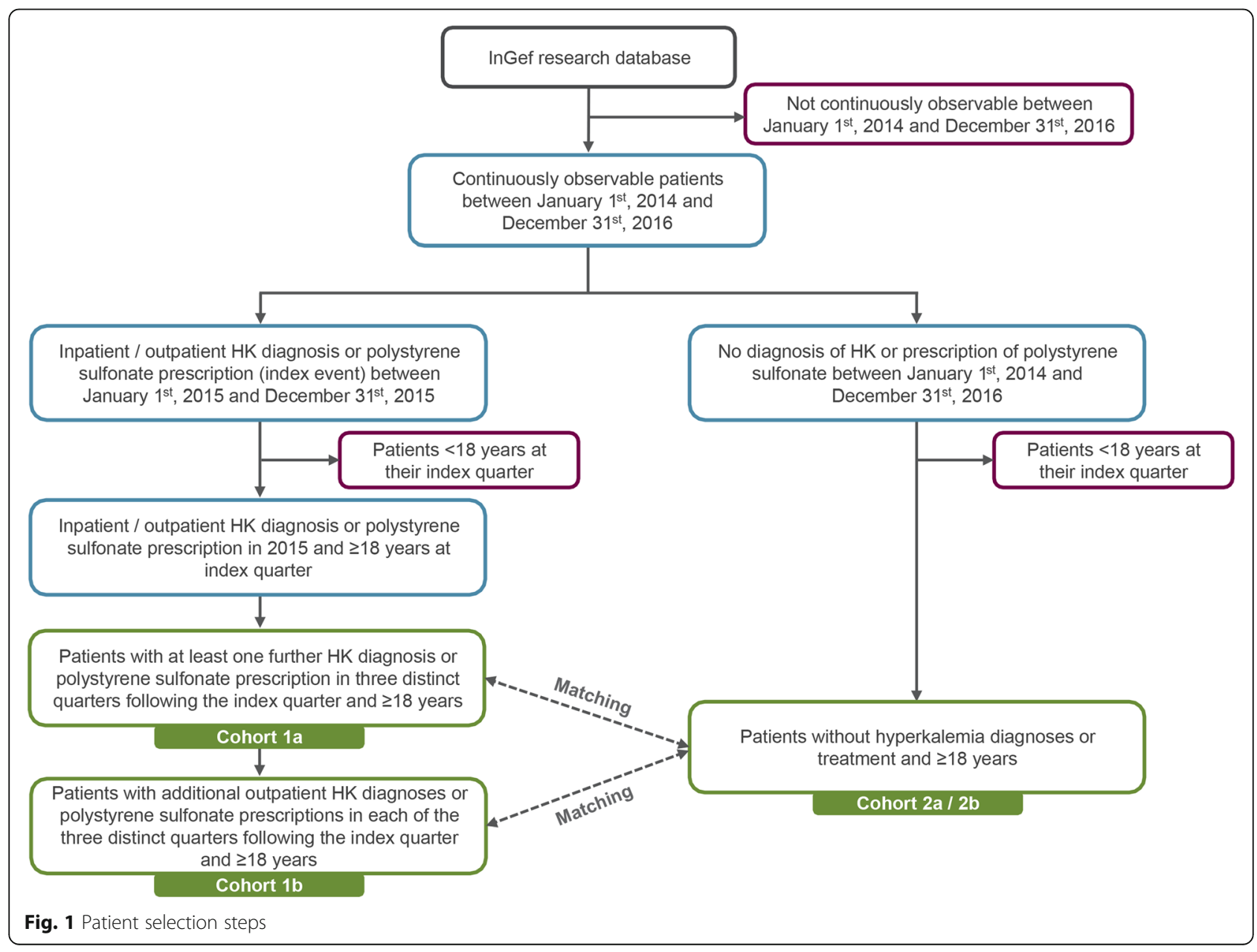


The matching performance was evaluated by calculating absolute standardized differences of the cohorts as well as by statistical testing.

\section{Outcome assessment}

The assessment of patient characteristics included demographics in the index quarter and disease-specific comorbidities, such as CKD, HF, and diabetes mellitus type 2 (T2DM) in the pre-index period. Further, the mean updated Charlson Comorbidity Index (CCI) was assessed for each HK cohort (see Additional file 1).

As the individual number of hospitalizations in the index quarter were taken into account as a matching parameter, the all-cause and HK-related hospitalizations were analyzed in the 3 quarters following the index quarter.

Concomitant treatment was determined for RAASi therapy using ATC codes. Furthermore, use of sodium or calcium polystyrene sulfonate (SPS/CPS) stratified by disease-specific comorbidities within the HK patient cohorts, taking the most severe diagnosis for the respective comorbidity (CKD/HF) into account, was assessed. Prescriptions were analyzed in terms of mean number of prescriptions for SPS/CPS and proportion of heavy users ( $\geq 8$ prescriptions in total or $\geq 2$ prescriptions per quarter) as well as prescribing physician for SPS/CPS in the post-index period.

Healthcare costs stratified by different healthcare sectors, such as inpatient, outpatient, pharmaceuticals, and remedies and aids were assessed in the post-index period.

Time to dialysis initiation was assessed using the Kaplan-Meier method for all HK patients suffering from CKD (all stages) who did not have dialysis in the preindex period. Therefore, the number of quarters between the first observable HK diagnosis and the occurrence of dialysis in the post index-up period was calculated. Patients were right censored in case of death, sickness fund switch or at the end of the observation period. Dialysis occurrence was assessed by OPS codes and defined EBM codes (see Additional file 1) at four possible timepoints (first quarter, second quarter, third quarter, fourth quarter) to analyze the time to event occurrence within the quarters of the follow-up period. Groups were compared with Log-Rank test.

Study outcomes were analyzed using counts and percentages and compared using the Chi-squared test for all categorical outcomes. Continuous outcomes were analyzed in terms of mean, standard deviation, 0.25 percentile, median, 0.75 percentile, minimum, maximum and sum and compared using the Wilcoxon signed rank test or the paired $t$ test depending on the distribution of the differences.

\section{Results}

Descriptive analysis

In total, $N=3333$ patients with non-acute outpatient treated HK (cohort 1a) and $N=1693$ chronic HK patients (cohort $1 \mathrm{~b}$ ) aged at least 18 years were identified in the database. Outpatient treated HK patients were 70.6 years old on average (SD: 13.3, range: $18-102$ ) and chronic HK patients were 71.2 years old on average (SD: 12.7, range: $21-101) .42 .6 \%$ of the non-acute outpatient treated HK patients and $43.9 \%$ of the chronic HK patients were female.

Almost one third of the patients in both cohorts did not have one of the defined disease-specific comorbidities or any combination thereof. Most nonacute outpatient treated (48.7\%) and chronic HK patients $(47.5 \%)$ had CKD stage 3,4 , or 5 in the preindex period. Diabetes mellitus type 2 (not subclassified) had a prevalence of $46.4 \%$ in the non-acute outpatient $\mathrm{HK}$ cohort and $45.7 \%$ in the chronic HK cohort, respectively. The prevalence of heart failure (all grades) alone, in combination with diabetes mellitus type 2 or CKD any stage was $20.4 \%$ in non-acute outpatient treated and $18.9 \%$ in chronic HK patients (see Table 1).

The analysis of concomitant medication within the individual post-index period of 4 quarters showed that $n=1824$ (54.7\%) of the non-acute outpatient treated HK patients (cohort 1a) and $n=927$ (54.8\%) chronic HK patients (cohort 1b) received at least one prescription of high-ceiling diuretics. Prescriptions for any agents of RAAS inhibitors were present in 1534 (46.0\%) and $760(44.9 \%)$ cohorts $1 \mathrm{a}$ and $1 \mathrm{~b}$, respectively. Patients with at least one prescription of SPS/ CPS accounted for $n=891$ patients (26.7\%) in cohort $1 \mathrm{a}$ and $n=356$ patients $(21.0 \%)$ in cohort $1 \mathrm{~b}$. Nephrologists prescribed $47.8 \%$ (1a) and $45.7 \%$ (1b) of SPS/CPS whereas prescriptions by cardiologists accounted for $0.3 \%$ (1a) and $0.3 \%$ (1b). The defined heavy use of SPS/CPS was detected in 54 (1.6\%) (1a) and $51(3.0 \%)$ (1b) (see Fig. 2).

$80.9 \%$ of the non-acute outpatient treated HK patients and $79.5 \%$ of the chronic HK patients who had at least one SPS/CPS prescription, also incurred a diagnosis of CKD (any stage) in the individual post-index period. A proportion of $35.6 \%$ (cohort 1a) and $27.2 \%$ (cohort 1b) suffered from CKD stage 5 and received dialysis, indicated by the respective coding and taking the most severe CKD diagnosis in the observation period into account

Moreover, 23.1\% of the outpatient treated HK patients as well as $18.5 \%$ of the chronic HK patients with at least one SPS/CPS prescription had diagnoses of heart failure with NYHA classes II to IV being the most prevalent NYHA classes (see Table 2). 
Table 1 Disease-specific comorbidities and CCI of HK patients

\begin{tabular}{|c|c|c|c|c|}
\hline \multirow[t]{2}{*}{ Disease (ICD-10-GM code) } & \multicolumn{2}{|c|}{$\begin{array}{l}\text { Non-acute outpatient treated HK patients } \\
\mathbf{N}=3333\end{array}$} & \multicolumn{2}{|c|}{$\begin{array}{l}\text { Chronic HK patients } \\
\boldsymbol{N}=1693\end{array}$} \\
\hline & $n$ & $\%$ & $\mathrm{n}$ & $\%$ \\
\hline \multicolumn{5}{|l|}{ CKD } \\
\hline CKD stages 1 to $5(\mathrm{~N} 18.1+\mathrm{N} 18.2+\mathrm{N} 18.3+\mathrm{N} 18.4+\mathrm{N} 18.5)$ & 1737 & 52.1 & 860 & 50.8 \\
\hline CKD stage 3 to 5 (N18.3 + N18.4 + N18.5) & 1623 & 48.7 & 805 & 47.5 \\
\hline \multicolumn{5}{|l|}{ HF } \\
\hline Heart failure (ANY) (I50.11-I50.14, I50.19) & 681 & 20.4 & 320 & 18.9 \\
\hline \multicolumn{5}{|l|}{ T2DM } \\
\hline Diabetes mellitus, type 2 (E11.-) & 1481 & 46.44 & 753 & 44.5 \\
\hline \multicolumn{5}{|l|}{ Comorbidities (CKD, T2DM, HF) } \\
\hline None & 943 & 28.3 & 483 & 28.5 \\
\hline One Comorbidity & 1203 & 36.1 & 633 & 37.4 \\
\hline CKD & 640 & 19.2 & 331 & 19.6 \\
\hline $\mathrm{T} 2 \mathrm{DM}$ & 486 & 14.6 & 264 & 15.6 \\
\hline $\mathrm{HF}$ & 77 & 2.3 & 38 & 2.2 \\
\hline Two Comorbidities & 865 & 26.0 & 431 & 25.5 \\
\hline$C K D+T 2 D M$ & 583 & 17.5 & 295 & 17.4 \\
\hline $\mathrm{CKD}+\mathrm{HF}$ & 192 & 5.8 & 88 & 5.2 \\
\hline $\mathrm{T} 2 \mathrm{DM}+\mathrm{HF}$ & 90 & 2.7 & 48 & 2.8 \\
\hline Three Comorbidities & 322 & 9.7 & 146 & 8.6 \\
\hline$\geq$ One Comorbidity & 2390 & 71.7 & 1210 & 71.5 \\
\hline \multicolumn{5}{|l|}{ Charlson Comorbidity Index (CCI) } \\
\hline CCl score: 0 & 397 & 11.9 & 185 & 10.9 \\
\hline CCl score: 1 & 502 & 15.1 & 283 & 16.7 \\
\hline CCl score: 2 & 485 & 14.6 & 247 & 14.6 \\
\hline CCl score: 3 & 494 & 14.8 & 265 & 15.7 \\
\hline CCl score: $\geq 4$ & 1455 & 43.7 & 713 & 42.1 \\
\hline Mean CCl score & 3.32 & & 3.26 & \\
\hline
\end{tabular}

\section{Matching analysis}

After matching the non-acute outpatient treated HK patients $\left(1 \mathrm{a}^{*}\right)$ to a non-HK cohort $\left(2 \mathrm{a}^{*}\right)$ and also matching the chronic HK patients $\left(1 b^{*}\right)$ to a non-HK cohort $\left(2 b^{*}\right)$, a study population of $n=3191$ patients with non-acute outpatient treated $\mathrm{HK}$ and $n=1664$ patients with chronic HK with respective control groups remained. After the exact matching, the aforementioned matching parameters were completely balanced with standardized differences of 0 and $p$-values of 1 for all factors (see Additional file 1) for the matching parameters before and after matching]. The mean number of hospitalizations in the index quarter was 0.5 (cohorts $\left.1 \mathrm{a}^{*} / 2 \mathrm{a}^{*}\right)$ and 0.4 (cohorts $1 b^{*} / 2 b^{*}$ ), respectively. Furthermore, half of the patients of all matched cohorts (50.0\% vs. $50.1 \%)$ did not have a CKD diagnosis and about $18 \%$ of the patients had a diagnosis of CKD stage 5 (see Supplementary Material for more detailed information).
Both HK cohorts showed a higher number of hospitalizations compared to non-HK patients (cohorts a*: 2732 vs. $1749 /$ cohorts $b^{*}: 1125$ vs. 930). HK patients had on average more hospitalizations than patients in the control group (cohorts $a^{*}: 0.9$ vs. $0.5, p \leq 0.001 /$ cohorts $b *$ : 0.7 vs. $0.6, p=0.002$ ). HK-related hospitalizations with HK as main diagnosis accounted for around $1 \%$ of the identified inpatient stays of HK patients.

The total number of dialysis events in the post-index period was highest in cohort $1 \mathrm{a}^{*}$ with 8324 events $(3132$ cohort 2a*), whereas cohort $1 b^{*}$ had 4602 events (1509 cohort $\left.2 b^{*}\right)$. The assessment of dialysis initiation in the one-year follow up revealed that individuals with $\mathrm{HK}$ had a twofold higher initiation rate than their matched counterparts. Further, the time to dialysis initiation was assessed with Kaplan-Meier (see Additional file 1 for the graphs), taking the occurrence of dialysis in each quarter into account. Individuals without hyperkalemia in the 

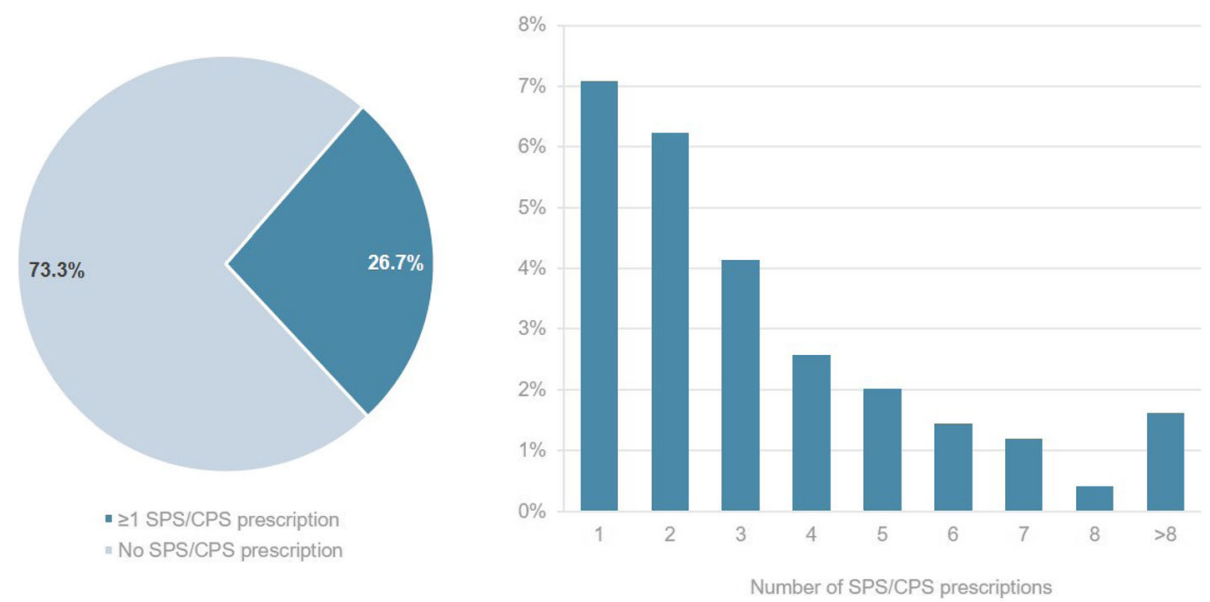

SPS/CPS prescriptions of chronic HK patients
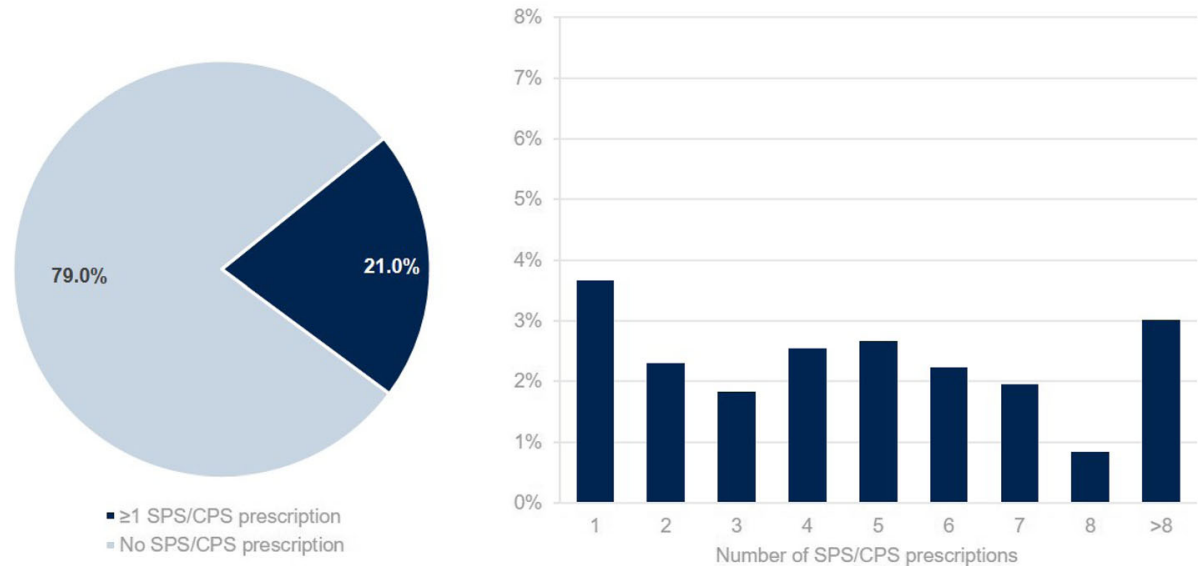

Fig. 2 Use of SPS/CPS in patients with non-acute outpatient treated and patients with chronic HK

respective cohorts had a higher probability of initiating dialysis treatment in the first four quarters than their matched hyperkalemia counterparts. Accordingly, the average time until dialysis initiation was higher for both hyperkalemia cohorts (cohort $1 \mathrm{a}^{*}$ mean in quarters 1.3 vs. cohort $2 \mathrm{a}^{*}$ mean in quarters 1.0 ; and cohort $1 \mathrm{~b}^{*}$ mean in quarters 1.0 vs. cohort $2 b^{*}$ mean in quarters 0.8 ;) (see Table 3).

The mean total healthcare costs in the post-index period of 1 year (4 quarters) were significantly higher for both HK cohorts and their matched counterparts (cohorts a*: $€ 17,076$ vs. $€ 11,301, p \leq 0.001 /$ cohorts $b^{*}: € 14$, 421 vs. $€ 11,606, p \leq 0.001)$. Additionally, when comparing cohort $1 \mathrm{a}^{*}$ and $2 \mathrm{a}$, significant differences in mean costs referring to the inpatient and outpatient care setting, as well as pharmaceuticals and devices and aids, could be reported. The observed higher costs in the chronic $\mathrm{HK}$ cohort $1 \mathrm{~b}^{*}$ were not significant for inpatient care costs and sick leave payment. It can be noted that the assessed population was on average over 70 years of age, therefore the sick leave costs were quite low. Significantly higher costs were observed in outpatient care, pharmaceuticals and devices and aids with differences in the mean costs (see Fig. 3). The costs of HK-related hospitalizations for inpatient care accounted for 29.2 and $20.7 \%$ for cohort $1 \mathrm{a}^{*}$ and $1 \mathrm{~b}$ * respectively.

\section{Discussion}

To our knowledge, this is the first retrospective claims data analysis assessing the burden and healthcare costs of non-acute outpatient treated HK and chronic HK in the German healthcare setting. The InGef research database utilized for this study is adjusted to represent the German population in terms of age and gender and further shows representativeness regarding hospitalizations, mortality rate, and prescriptions which was validated by 
Table 2 Disease-specific comorbidities of patients with and without SPS/CPS use

\begin{tabular}{|c|c|c|c|c|c|c|c|c|}
\hline & \multicolumn{4}{|c|}{$\begin{array}{l}\text { Non-acute outpatient treated HK patients (cohort 1a) } \\
(\boldsymbol{N}=3333)\end{array}$} & \multicolumn{4}{|c|}{$\begin{array}{l}\text { Chronic HK patients (cohort 1b) } \\
(\boldsymbol{N}=1693)\end{array}$} \\
\hline & \multicolumn{2}{|c|}{$\begin{array}{l}\text { Patients with } \geq 1 \mathrm{SPS} / \mathrm{CPS} \\
\text { prescription }\end{array}$} & \multicolumn{2}{|c|}{$\begin{array}{l}\text { Patients without SPS/CPS } \\
\text { prescription }\end{array}$} & \multicolumn{2}{|c|}{$\begin{array}{l}\text { Patients with } \geq 1 \text { SPS/CPS } \\
\text { prescription }\end{array}$} & \multicolumn{2}{|c|}{$\begin{array}{l}\text { Patients without SPS/CPS } \\
\text { prescription }\end{array}$} \\
\hline & $n$ & $\%$ & $n$ & $\%$ & $n$ & $\%$ & $\mathrm{n}$ & $\%$ \\
\hline \multicolumn{9}{|c|}{ Chronic kidney disease (most severe diagnosis coding was taken into account) } \\
\hline CKD stage $1 \& 2$ & 12 & 1.3 & 102 & 4.2 & $<5$ & NA & 51 & 3.8 \\
\hline CKD stage 3 & 139 & 15.6 & 395 & 16.2 & 59 & 16.6 & 193 & 14.4 \\
\hline CKD stage 4 & 198 & 22.2 & 245 & 10.0 & 102 & 28.7 & 134 & 10.0 \\
\hline CKD stage 5 without dialysis & 55 & 6.2 & 63 & 2.6 & 21 & 5.9 & 42 & 3.1 \\
\hline CKD stage 5 with dialysis & 317 & 35.6 & 211 & 8.6 & 97 & 27.2 & 157 & 11.7 \\
\hline CKD (distinct) & 721 & 80.9 & 1016 & 41.6 & 283 & 79.5 & 577 & 43.2 \\
\hline \multicolumn{9}{|c|}{ Heart failure (most severe diagnosis coding was taken into account) } \\
\hline Heart failure NYHA class I & 14 & 1.6 & 26 & 1.1 & $<5$ & NA & 17 & 1.3 \\
\hline Heart failure NYHA class II & 51 & 5.7 & 108 & 4.4 & 21 & 5.9 & 61 & 4.6 \\
\hline Heart failure NYHA class III & 57 & 6.4 & 149 & 6.1 & 16 & 4.5 & 88 & 6.6 \\
\hline Heart failure NYHA class IV & 63 & 7.1 & 136 & 5.6 & 19 & 5.3 & 64 & 4.8 \\
\hline Heart failure unspecified & 21 & 2.4 & 56 & 2.3 & 6 & 1.7 & 24 & 1.8 \\
\hline Heart failure (distinct) & 206 & 23.1 & 475 & 19.5 & 66 & 18.5 & 254 & 19.0 \\
\hline
\end{tabular}

a recent study [16]. Health insurance in Germany is statutory and covers almost $85 \%$ of the population and the contents of reimbursement are set in social law, offering the same comprehensive benefit package across individual statutory health insurances [17]. Therefore, the study results can be considered reliable in the context of German SHI real world evidence.

It is often stated that hyperkalemia is most commonly associated with diseases such as CKD, DM or HF. But little is known about the prevalence of HK in general or as a comorbidity of one of the aforementioned diseases. A Swedish observational study revealed an incidence of hyperkalemia of $7 \%$ within adult individuals [2]. Another study published by Einhorn and colleagues [13] compared the occurrence of hyperkalemia between patients with and without CKD in veteran patients with at least one hospitalization and one potassium measurement during a 1 year time period. The prevalence of $\mathrm{HK}$ in patients with any stage of CKD was $26.6 \%$ whereas only $8.9 \%$ of patients without chronic kidney disease showed potassium values of $\geq 5.5 \mathrm{mEq} / \mathrm{L}$. In the same study, the proportion of patients with hyperkalemia for the whole study population $(13.7 \%)$, patients with diabetes $(19.5 \%)$ and who received ACE inhibitors and/or ARB (16.9\%) where stated.

In this analysis, HK was the primary selection criterion to identify patients with non-acute outpatient treated or chronic HK and comorbidities were assessed for these cohorts in a second step. It was shown that more than $70 \%$ of patients in both cohorts suffered from at least one condition of CKD, HF or T2DM and that the proportions were higher in patients receiving at least one prescription of SPS/CPS especially for CKD. The use of this HK-specific medication was rather low with $26.6 \%$ of patients with at least one prescription in cohort $1 \mathrm{a}$ and $20.9 \%$ in cohort $1 \mathrm{~b}$.

Table 3 Time-to-dialysis initiation event

\begin{tabular}{|c|c|c|c|c|c|c|c|}
\hline & \multicolumn{2}{|c|}{$\begin{array}{l}\text { No. of patients with dialysis } \\
\text { initiation event }\end{array}$} & \multicolumn{5}{|c|}{$\begin{array}{l}\text { Average length of observation time until dialysis } \\
\text { initiation event (in quarters) }\end{array}$} \\
\hline & $\mathrm{n}$ & $\%$ & Mean & STD & Median & Min & Max \\
\hline Patients with outpatient hyperkalemia (cohort $1 \mathrm{a}^{*}$ ) & 118 & 10.5 & 1.3 & 1.2 & 1.0 & 0.0 & 3.0 \\
\hline Patients without hyperkalemia (cohort $2 a^{*}$ ) & 62 & 4.8 & 1.0 & 1.1 & 1.0 & 0.0 & 3.0 \\
\hline Patients with chronic hyperkalemia (cohort $1 b^{*}$ ) & 51 & 8.7 & 1.0 & 1.2 & 1.0 & 0.0 & 3.0 \\
\hline Patients without hyperkalemia (cohort $2 b^{*}$ ) & 28 & 4.2 & 0.8 & 1.0 & 0.0 & 0.0 & 3.0 \\
\hline
\end{tabular}




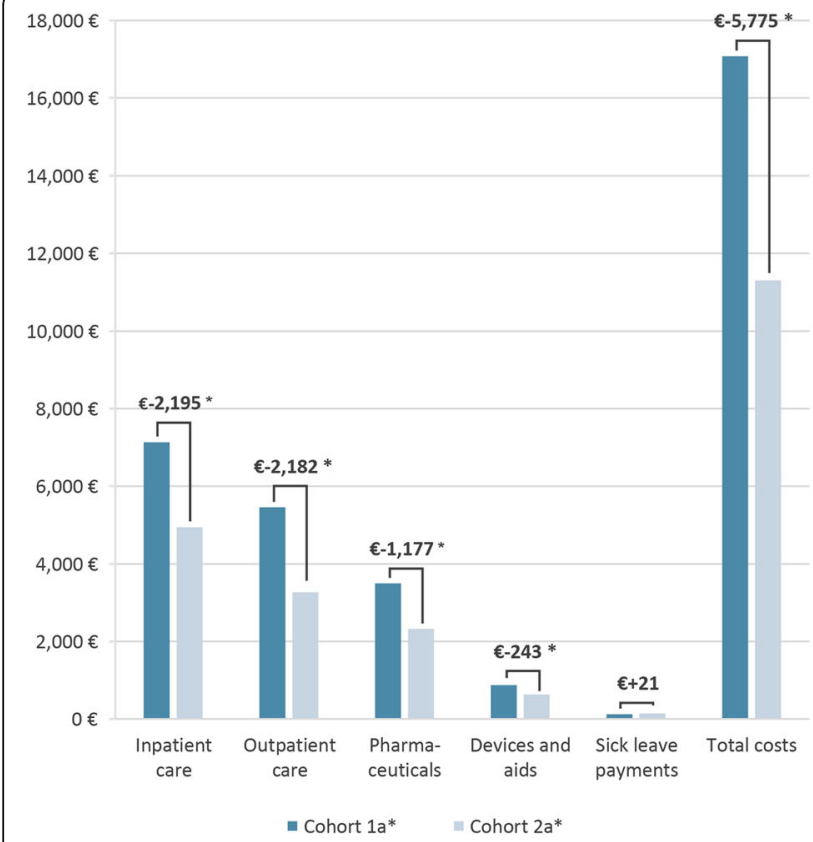

Fig. 3 Mean healthcare costs of the matched HK and non-HK cohorts

Furthermore, the results of the analysis showed that the number of patients with dialysis initiation was significantly higher in both HK cohorts than in the nonHK cohorts. Non-acute outpatient treated HK patients (cohort 1a) had the highest total number of dialysis events, while the mean number of dialysis events was highest in patients with chronic HK (cohort 1b) who had an average number of 90 dialysis events in the individual post-index period. Taking these results into account, $\mathrm{HK}$ is associated with a higher risk of dialysis initiation and the group of patients suffering from chronic HK have even more dialysis events than the non-acute outpatient treated HK patients. However, it should be noted, that individuals were censored upon the occurrence of death, therefore, individuals not deceasing might have had a longer time period under risk. An Italian prospective study showed that, in addition, the likelihood for end stage renal disease (ESRD) and, therefore, the need for dialysis increases with a higher level of serum potassium [18].

Hyperkalemia was determined as a predictor of total costs in patients receiving RAASi treatment for all disease cohorts of chronic kidney disease, heart failure and diabetes by Epstein et al. [19]. For patients with chronic kidney disease and heart failure it was recently shown by Polson and colleagues [20], that hyperkalemia is linked to higher medical costs in patients with and without RAASi treatment. In an analysis by Betts and colleagues [11] for HK in the US the average all-cause costs added up to $\$ 15,983$. The results of this analysis are in line with the aforementioned publications and show that

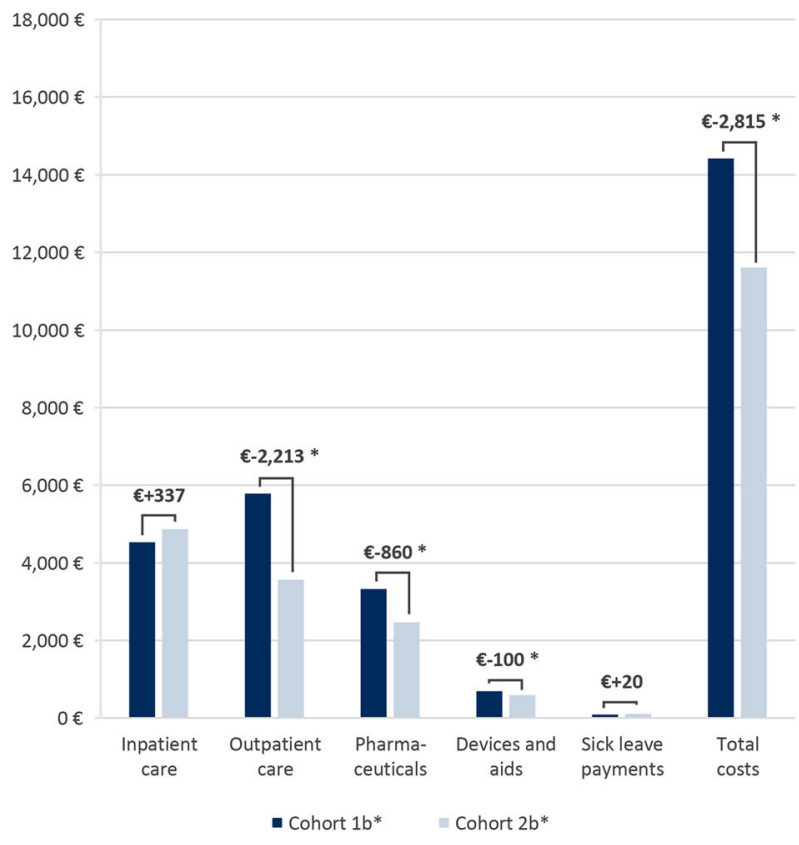

especially non-acute outpatient treated hyperkalemia patients show significantly higher total healthcare costs and that hyperkalemia related hospitalizations account for more than $20 \%$ of inpatient care costs in both cohorts.

\section{Limitations}

In general, claims data are considered an appropriate source to explore and analyze health-relevant real world evidence queries related to, but not limited to, epidemiology and burden of illness, as this data is recorded for reimbursement purposes and not connected to the purpose of the respective study, offering the possibility of less selection bias. Nevertheless, there are limitations related to the use of claims data which need to be considered when interpreting the study results. Firstly, claims data are collected for the purpose of accounting and unless data linkage is performed, no clinical data is available. In this context, the unavailability of acid-base status or the adoption of a low potassium diet should be taken into account when interpreting the results, as the definition of hyperkalemia is more nuanced and cannot be fully addressed without additional clinical information. Further, the results should be considered with the background of the anticipated coding quality and the need for the respective individuals to seek treatment. Additionally, diagnoses in the outpatient setting are coded on a quarterly basis and cannot be attributed to an exact date. The private sector was not covered by this analysis, as the data pool only includes sickness funds 
from the SHI system. Concerning the performed matching, unobserved variables may be unequally distributed in the groups, which might lead to potentially biased results.

\section{Conclusions}

To date, hyperkalemia prevalence has been assessed by analyzing overall comorbid conditions, such as CKD and DM, and reporting the comorbid occurrence of hyperkalemia. Within this study, the prevalence of hyperkalemia was the main focus irrespective of any comorbid diseases. However, our analysis revealed that HK patients are multi-morbid and present with an increased prevalence of CKD and DM, which has previously been shown in other analyses.

Given the increased disease burden and the associated healthcare costs of patients with non-acute outpatient treated or chronic HK, in comparison to their matched counterparts, a necessity for treatment improvement could be suggested. On the other hand, the use of the only HK-specific medication (SPS / CPS) that was available during the study period, was rather low.

A possible conclusion is that an adequate treatment of HK could reduce the patients' hospitalizations, the need for dialysis and in turn the healthcare costs from a long term perspective, which warrants further research.

\section{Supplementary information}

Supplementary information accompanies this paper at https://doi.org/10 1186/s12882-020-01942-2.

Additional file 1. Matching parameters before and after matching Description of data: Matching variables included in the exact 1:1 matching for non-acute outpatient HK vs. non-HK patients and for acute HK vs. non-HK patients. Coding lists including dialysis, hyperkalemia, RAASi, CKD stages, heart failure, and diabetes mellitus identification. Charlson Comorbidity Index overview. Time to dialysis initiation (Kaplan Meier curves)

\section{Abbreviations \\ ACE: Angiotensin-converting enzyme; ARB: Angiotensin receptor blockers; ATC: Anatomical therapeutic chemical classification system; CKD: Chronic kidney disease; CPS: Calcium polystyrene sulfonate; CVD: Cardiovascular disease; DESTATIS: Statistisches Bundesamt (Federal Office of Statistics); DM: Diabetes mellitus; EBM: Einheitlicher Bewertungsmassstab (Practitioners' Fee Scale within the Statutory Health Insurance Scheme); ESRD: End stage renal disease; GFR: Glomerular filtration rate; HF: Heart failure; HK: Hyperkalemia; ICD-10-GM: International Statistical Classification of Diseases and Related Health Problems, 10th revision, German Modification; InGef: "Institut fuer angewandte Gesundheitsforschung Berlin" (Institute for applied health research, Berlin); NYHA: New York Heart Association; OPS: Operationen- und Prozedurenschluessel (Key of operations and procedures); Q: Quarter; RAASi: Renin-angiotensin-aldosterone system inhibitors; SD: Standard deviation; SHI: Statutory Health System; SPS: Sodium polystyrene sulfonate; T2DM: Type 2 diabetes mellitus}

\section{Acknowledgements}

The analyses were performed in collaboration with Prof. Dr. Wolfgang Greiner and the Institut für angewandte Gesundheitsforschung (InGef).

\section{Authors' contributions}

JSH, KSK, CM, TH, IB and SB were involved in the development of the study design, interpretation of the data and drafting the manuscript. Furthermore JSH, KSK, CM, TH, IB and SB interpreted the data, critically revised the manuscript, gave final approval of the version to be published and agreed to be held accountable for all aspects of the publication.

\section{Funding}

This study was funded by Vifor Pharma Deutschland $\mathrm{GmbH}$ and executed by Xcenda $\mathrm{GmbH}$. Vifor Pharma Deutschland $\mathrm{GmbH}$ and Xcenda $\mathrm{GmbH}$ were involved in all stages of the study, including study design, analysis, interpretation of the analysis results and writing the manuscript.

\section{Availability of data and materials}

All data generated or analyzed during this study are included in this published article [and its supplementary information files]. The data that support the findings of this study are not publicly available as they are owned by the statutory health insurances. Data requests need to be granted by the InGef and the participating health insurances.

\section{Ethics approval and consent to participate}

Not applicable. No ethical approval and consent was necessary as this is a retrospective database study based on anonymized claims data from the Institut für angewandte Gesundheitsforschung Berlin (InGef) research database.

\section{Consent for publication}

Not applicable.

\section{Competing interests}

$\mathrm{TH}$ and IB are employees of Vifor Pharma GmbH.

JSH, KSK, CM and SB are employees of Xcenda, which has a research consultancy agreement with Vifor Pharma GmbH.

The data analysis was performed in collaboration with Prof. Dr. Wolfgang Greiner and the Institut für angewandte Gesundheitsforschung Berlin (InGef).

\section{Author details}

${ }^{1}$ Xcenda GmbH, Lange Laube 31, 30159 Hannover, Germany. ${ }^{2}$ Vifor Pharma Gruppe, Pharma Deutschland GmbH, Baierbrunner Str. 29, 81379 Munich, Germany.

Received: 20 May 2019 Accepted: 12 July 2020

Published online: 08 August 2020

\section{References}

1. Evans K, Greenberg A. Hyperkalemia: a review. J Intensive Care Med. 2005; 20(5):272-90.

2. Nilsson E, Gasparini A, Ärnlöv J, Xu H, Henriksson K, Coresh J, et al. Incidence and determinants of hyperkalemia and hypokalemia in a large healthcare system. Int J Cardiol. 2017;245:277-84.

3. Elliot M, Ronksley P, Clase C, Ahmed S, Hemmelgarn B. Management of patients with acute hyperkalemia. CMAJ. 2010;182(15):1631-5.

4. Stevens M, Dunlay R. Hyperkalemia in hospitalized patients. Int Urol Nephrol. 2000;32(2):177-80.

5. Raebel M. Hyperkalemia associated with use of angiotensin-converting enzyme inhibitors and angiotensin receptor blockers. Cardiovasc Ther. 2012;30:e156-66.

6. Kim H, Han S. Therapeutic approach to hyperkalemia. Nephron. 2002; 92(Suppl 1):33-40.

7. Michel A, Martín-Pérez M, Ruigómez A, Rodríguez LG. Risk factors for hyperkalaemia in a cohort of patients with newly diagnosed heart failure: a nested case-control study in UK general practice. Eur J Heart Fail. 2015;17(2):205-13.

8. Perazella M. Drug-induced hyperkalemia: old culprits and new offenders. Am J Med. 2000;109(4):307-14

9. Saito $Y$, Yamamoto $H$, Nakajima H, Takahashi O, Komatsu Y. Incidence of and risk factors for newly diagnosed hyperkalemia after hospital discharge in non-dialysis-dependent CKD patients treated with RAS inhibitors. PLOS One. 2017;12(9):e0184402.

10. Kovesdy C. Epidemiology of hyperkalemia: an update. Kidney Int Suppl. 2011;6(1):3-6

11. Betts K, Woolley J, Mu F, Xiang C, Tang W, Wu E. The cost of hyperkalemia in the United States. Kidney Int Rep. 2018;3(2):385-93. 
12. Drawz P, Babineau D, Rahman M. Metabolic complications are common in elderly patients with chronic kidney disease. J Am Geriatr Soc. 2012;60(2):310-5.

13. Einhorn L, Zhan M, Hsu V, Walker L, Moen M, Seliger S, et al. The frequency of hyperkalemia and its significance in chronic kidney disease. Arch Int Med. 2009;169(12):1156-62.

14. Weisberg L. Management of severe hyperkalemia. Crit Care Med. 2008; 36(12):3246-51.

15. Fried L, Kovesdy C, Palmer B. New options for the management of chronic hyperkalemia. Kidney Int Suppl. 2017;7(3):164-70.

16. Andersohn F, Walker J. Characteristics and external validity of the German health risk institute (HRI) database. Pharmacoepidemiol Drug Saf. 2016;25(1):106-9.

17. Gesundheit BF. Kennzahlen der Gesetzlichen Krankenversicherung 2005 bis 2018; 2018.

18. Provenzano M, Minutolo R, Chiodini P, Bellizzi V, Nappi F, Russo D, et al. Competing-risk analysis of death and end stage kidney disease by Hyperkalaemia status in non-Dialysis chronic kidney disease patients receiving stable nephrology care. J Clin Med. 2018;7(499):1-13.

19. Epstein M, Alvarez P, Reaven N, Funk S, McGaughey K, Brenner M, et al. Evaluation of clinical outcomes and costs based on prescribed dose level of renin-angiotensinaldosterone system inhibitors. Am J Manag Care. 2016;22(11):S311-24.

20. Polson M, Lord T, Kangethe A, Speicher L, Farnum C, Brenner M, et al. Clinical and economic impact of hyperkalemia in patients with chronic kidney disease and heart failure. J Manag Care Spec Pharm. 2017;23(4-a Suppl):S2-9.

\section{Publisher's Note}

Springer Nature remains neutral with regard to jurisdictional claims in published maps and institutional affiliations.

Ready to submit your research? Choose BMC and benefit from:

- fast, convenient online submission

- thorough peer review by experienced researchers in your field

- rapid publication on acceptance

- support for research data, including large and complex data types

- gold Open Access which fosters wider collaboration and increased citations

- maximum visibility for your research: over $100 \mathrm{M}$ website views per year

At $\mathrm{BMC}$, research is always in progress.

Learn more biomedcentral.com/submissions 